\title{
Quantum decoherence in finite size exciton-phonon systems
}

\author{
Vincent Pouthier* \\ Institut UTINAM, Université de Franche-Comté, \\ CNRS UMR 6213, 25030 Besançon Cedex, France
}

(Dated: January 26, 2011)

\begin{abstract}
Based on the operatorial formulation of the perturbation theory, the properties of a confined exciton coupled with phonons in thermal equilibrium is revisited. Within this method, the dynamics is governed by an effective Hamiltonian which accounts for exciton-phonon entanglement. The exciton is dressed by a virtual phonon cloud whereas the phonons are clothed by virtual excitonic transitions. Special attention is thus paid for describing the time evolution of the excitonic coherences at finite temperature. As in an infinite lattice, temperature-enhanced quantum decoherence takes place. However, it is shown that the confinement softens the decoherence. The coherences are very sensitive to the excitonic states so that the closer to the band center the state is located, the slower the coherence decays. In particular, for odd lattice sizes, the coherence between the vacuum state and the one-exciton state exactly located at the band center survives over an extremely long time scale. A superimposition involving the vacuum and this specific one-exciton state behaves as an ideal qubit insensitive to its environment.
\end{abstract}

PACS numbers:

\section{INTRODUCTION}

Molecular lattices show regularly distributed atomic subunits along which the energy of a specific electronic transition, or a high frequency vibrational mode, delocalizes due to dipole-dipole interaction. This gives rise to a narrow-band exciton ${ }^{1-3}$ whose quantum states are superimpositions of local states and which is able to move coherently along the lattice. However, the exciton interacts with the phonons of the host medium ${ }^{4-6}$. They induce stochastic modulations of the local state energies and they tend to break the excitonic coherence.

The exciton-phonon system is a prototype of an open system (exciton) coupled with a thermal bath (phonons) whose characterization is essential in understanding many phenomena: exciton dynamics in photosynthetic antenna and polymers ${ }^{7-11}$, vibron propagation in $\alpha$-helices ${ }^{12-19}$ and adsorbed nanostructures ${ }^{20-25}$, vibrational qubits in quantum channels ${ }^{26,27}$. The exciton properties are thus encoded in the reduced density matrix (RDM) whose behavior is governed by a generalized master equation $(\mathrm{GME})^{28,29}$. Among the different strategies elaborated to derive a GME, the time-convolutionless approach (TCL) plays a central role $\mathrm{e}^{29-34}$. It provides a time local GME in which the phonon influence is encoded in a time-dependent relaxation operator. It is particularly suitable for describing non-markovian processes and for deriving approximate GME. The Born approximation is often invoked resulting in a second order expansion $\left(\mathrm{TCL}_{2}\right)$ with respect to the exciton-phonon coupling.

In a recent series of papers ${ }^{35-39}$, special attention has been paid for studying a narrow-band exciton coupled with acoustic phonons. Assuming that the exciton propagates slower than the phonons, the nonadiabatic weak coupling limit has been considered (see for instance Refs. ${ }^{40-42}$ and the references inside). Therefore, the $\mathrm{TCL}_{2}$ method has been used for describing the evolution of the excitonic coherences. These coherences measuring the ability of the exciton to develop superimpositions involving the vacuum and one-exciton states, they are required to characterize various processes (optical response ${ }^{43}$, quantum state transfer $\left.{ }^{44} \ldots\right)$. Our studies have revealed that the behavior of the coherences strongly depends on the lattice size.

In an infinite lattice ${ }^{35,36}$, the phonons behave as a reservoir and the Born approximation is legitimate. In the nonadiabatic limit, the Markov limit is reached and the relaxation operator becomes time independent. Dephasing limited-coherent dynamics takes place so that the coherences irreversibly decrease with time. They localize and do not propagate significantly along the lattice $^{36}$. By contrast, in a finite size lattice, a strong non-markovian regime takes place due to the discrete nature of the phonon energy spectrum ${ }^{37,38}$. The confinement favors quantum recurrences that provide to the relaxation operator an almost periodic nature which mainly results from the coupling with the lowest frequency phonon mode (LFPM). The GME reduces to a linear system of differential equations with almost periodic coefficients. Therefore, parametric resonances between specific excitonic frequencies and LFPM give rise to an exponential growth of the RDM indicating that the $\mathrm{TCL}_{2}$ method breaks down ${ }^{38}$.

To overcome this difficulty, finite size effects have been revisited within the standard perturbation theory $(\mathrm{PT})^{39}$. To proceed, the system "exciton+LFPM" has been considered. Due to its simplicity, it was solved exactly so that the PT performance has been checked. It has been shown that PT is a powerful tool for describing the spectral properties of the system. Furthermore, contrary to $\mathrm{TCL}_{2}$, PT is particularly suitable for characterizing the coherence dynamics. Quite naturally, the present paper is thus devoted to the generalization of PT to include the influence of all the phonon modes. However, we shall consider the operatorial formulation of PT that in- 
volves a perturbative unitary transformation ${ }^{45}$. Within this method, the exciton-phonon system is governed by an effective Hamiltonian that accounts for excitonphonon entanglement. It is no longer necessary to derive the perturbed eigenstates to evaluate the coherences, the required information being encoded in the transformation.

The paper is organized as follows. In Sec. II, the exciton-phonon Hamiltonian is described. Then, PT is applied to build the transformation and to derive the effective Hamiltonian. An approximate expression for the coherences is finally established. In Sec. III, the coherence dynamics is studied numerically and the results are discussed in Sec. IV.

\section{THEORETICAL BACKGROUND}

\section{A. Model Hamiltonian}

In a confined lattice with $N$ sites $x=1, \ldots, N$, oneexciton states are the first excited states of $N$ coupled two-level systems ${ }^{35-39}$. They correspond to $N$ superimpositions of incident and reflected plane waves with quantized wave vectors $K_{k}=k \pi / L$, with $k=1, . ., N$ and $L=N+1$. The $k$ th eigenstate is defined as

$$
|k\rangle=\sum_{x=1}^{N} \sqrt{\frac{2}{L}} \sin \left(K_{k} x\right)|x\rangle,
$$

where $|x\rangle$ is the first excited state of the $x$ th two-level system. The discrete eigenenergies $\omega_{k}=\omega_{0}+2 \Phi \cos \left(K_{k}\right)$ belong to a band centered on the Bohr frequency $\omega_{0}$ of each two-level system. Its bandwidth $4 \Phi$ involves the exciton hopping constant $\Phi$ between neighboring sites ( $\hbar=1$ will be used throughout this paper). The exciton Hamiltonian is thus defined as : $H_{A}=\sum_{k=1}^{N} \omega_{k}|k\rangle\langle k|$. Note that the vacuum state $|\oslash\rangle$ describing all the twolevel systems in their ground state with zero energy, the dimension of the exciton Hilbert space $\mathcal{E}_{A}$ is equal to $N+1$. The phonons are the elementary excitations associated to the external motions of the lattice sites that behave as point masses $M$ connected via force constants $W$. They refer to $N$ normal modes with wave vectors $Q_{p}=p \pi / L$ and frequencies $\Omega_{p}=\Omega_{c} \sin \left(Q_{p} / 2\right)$, with $p=1, . ., N$ and $\Omega_{c}=\sqrt{4 W / M}$. In the phonon Hilbert space $\mathcal{E}_{B}$, the dynamics is governed by the Hamiltonian $H_{B}=\sum_{p=1}^{N} \Omega_{p} a_{p}^{\dagger} a_{p}, a_{p}^{\dagger}$ and $a_{p}$ being standard phonon operators. Finally, the exciton-phonon coupling $V$ accounts for the modulation of each two-level system Bohr frequency induced by the lattice vibrations as (for more details see Ref. ${ }^{38}$ )

$$
V=\sum_{p=1}^{N} M_{p}\left(a_{p}^{\dagger}+a_{p}\right)
$$

where the operator $M_{p}$ acts in $\mathcal{E}_{A}$, only. It is defined as

$$
M_{p}=\sum_{k=1}^{N} \sum_{k^{\prime}=1}^{N} \eta_{p} S_{p k k^{\prime}}|k\rangle\left\langle k^{\prime}\right| .
$$

where $\eta_{p}=\left[\left(E_{B} \Omega_{p} / L\right)\left(1-\left(\Omega_{p} / \Omega_{c}\right)^{2}\right)\right]^{1 / 2}$ measures the coupling strength with the $p$ th phonon mode, $E_{B}$ being the small polaron binding energy. In Eq.(3), $S_{p k k^{\prime}}$ is written as

$$
S_{p k k^{\prime}}=\delta_{p, k-k^{\prime}}+\delta_{p, k^{\prime}-k}-\delta_{p, k+k^{\prime}}-\delta_{p, 2 L-k-k^{\prime}} .
$$

The finite size exciton-phonon system is governed by the Hamiltonian $H=H_{0}+V$ where $H_{0}=H_{A}+H_{B}$ is the unperturbed Hamiltonian. Since $H$ conserves the exciton number, the Hilbert space $\mathcal{E}=\mathcal{E}_{A} \otimes \mathcal{E}_{B}$ is partitioned into independent subspaces as $\mathcal{E}=\mathcal{E}_{0} \oplus \mathcal{E}_{1}$. In the zero-exciton subspace $\mathcal{E}_{0}, V=0$ so that the unperturbed states are eigenstates of $H$. They correspond to tensor products involving the vacuum $|\oslash\rangle$ and the phonon number states $\left|\left\{n_{p}\right\}\right\rangle=\left|n_{1}, \ldots, n_{N}\right\rangle$. They describe $n_{p}$ free phonons with energy $n_{p} \Omega_{p}, p=1, \ldots, N$. In the oneexciton subspace $\mathcal{E}_{1}$, the unperturbed states $|k\rangle \otimes\left|\left\{n_{p}\right\}\right\rangle$ refer to free phonons accompanied by an exciton in state $|k\rangle$. Since $V$ turns on in $\mathcal{E}_{1}$, they are no longer eigenstates of $H$. The exact eigenstates are entangled excitonphonon states that result from scattering processes which mix both exciton and phonon degrees of freedom. Indeed, $V$ yields exciton scattering from $|k\rangle$ with energy $\omega_{k}$, to $\left|k^{\prime}\right\rangle$ with energy $\omega_{k^{\prime}}$, via the exchange of a phonon $p$ with energy $\Omega_{p}$. The allowed transitions are specified by the rules $S_{p k k^{\prime}} \neq 0$ that generalize the concept of momentum conservation in a finite size lattice.

The key point it that these allowed transitions do not conserve the energy within the nonadiabatic limit, as in infinite lattices ${ }^{46-48}$. Provided that $4 \Phi<\Omega_{c}$, there is no resonance between coupled unperturbed states since $\omega_{k}-\omega_{k^{\prime}} \neq \pm \Omega_{p}$. Consequently, within the weak coupling limit $\left(E_{B} \ll \Phi\right)$, second order PT can be applied to treat the influence of the coupling $V^{39}$.

\section{B. Perturbation theory}

The operatorial formulation of $\mathrm{PT}^{45}$ is detailed in Appendix A. It is based on the introduction of a unitary transformation $U$ that diagonalizes the Hamiltonian $\hat{H}=U H U^{\dagger}$ in the unperturbed basis. The key point it that $U$ is expanded as a Taylor series with respect to $V$ so that the diagonalization is obtained at a given order. After simple algebraic manipulations, the transformed Hamiltonian up to second order is written as $\hat{H}=\hat{H}_{A}+\sum_{k=1}^{N} \hat{H}_{B}^{(k)} \otimes|k\rangle\langle k|\left(\right.$ in $\left.\mathcal{E}_{1}\right)$, where

$$
\begin{aligned}
\hat{H}_{A} & =\sum_{k=1}^{N}\left(\omega_{k}+\delta \omega_{k}\right)|k\rangle\langle k| \\
\hat{H}_{B}^{(k)} & =\sum_{p=1}^{N}\left(\Omega_{p}+\delta \Omega_{p k}\right) a_{p}^{\dagger} a_{p} .
\end{aligned}
$$


The contribution $\hat{H}_{A}$ is the effective exciton Hamiltonian. It reveals that in state $|k\rangle$, the energy of an exciton $\hat{\omega}_{k}=\omega_{k}+\delta \omega_{k}$ is renormalized due to its coupling with the phonons. The correction $\delta \omega_{k}$ is defined as (Eq.(A8))

$$
\delta \omega_{k}=\sum_{p=1}^{N} \sum_{k^{\prime}=1}^{N} \frac{\left\langle k\left|M_{p}\right| k^{\prime}\right\rangle^{2}}{\omega_{k}-\omega_{k^{\prime}}-\Omega_{p}} .
$$

It results from the spontaneous emission of a phonon $p$ during which the exciton realizes a transition from $|k\rangle$ to $\left|k^{\prime}\right\rangle$. However, in the nonadiabatic limit, the energy is not conserved during the transition so that the phonon emission is not a real process. The exciton is only able to exchange a virtual phonon which is first emitted and then immediately reabsorbed. In accordance with the small polaron concept, $\delta \omega_{k}$ is the manifestation of the so-called dressing effect. The exciton does no longer propagate freely but it is dressed by a virtual phonon cloud.

Similarly, when the exciton occupies a state $|k\rangle$, the phonon dynamics is governed by an effective Hamiltonian $\hat{H}_{B}^{(k)}$ (Eq.(5)). Each phonon of the $p$ th mode experiences an energy shift $\delta \Omega_{p k}$ expressed as (Eq.(A8))

$$
\delta \Omega_{p k}=\sum_{k^{\prime}=1}^{N} \frac{2\left\langle k\left|M_{p}\right| k^{\prime}\right\rangle^{2}\left(\omega_{k}-\omega_{k^{\prime}}\right)}{\left(\omega_{k}-\omega_{k^{\prime}}\right)^{2}-\Omega_{p}^{2}} .
$$

The correction $\delta \Omega_{p k}$ results from two mechanisms. First, the phonon $p$ can be absorbed giving rise to excitonic transitions from $|k\rangle$ to $\left|k^{\prime}\right\rangle$. Such a process does not conserve the energy within the nonadiabatic limit so that the phonon is immediately re-emitted. Second, the phonon $p$ can induce the stimulated emission of a second phonon during which the exciton realizes transitions. But, as previously, the emitted phonon is immediately reabsorbed. Both mechanisms are virtual processes indicating that the phonons do no longer evolve freely but are dressed by virtual excitonic transitions.

The operator $\hat{H}$ is the effective exciton-phonon Hamiltonian. Being diagonal in the unperturbed basis, its eigenvalues define the system eigenenergies up to second order in $V$ as $\epsilon_{k,\left\{n_{p}\right\}}=\hat{\omega}_{k}+\sum_{p=1}^{N} n_{p}\left(\Omega_{p}+\delta \Omega_{p k}\right)$. The corresponding eigenstates are defined as $\left|\Psi_{k,\left\{n_{p}\right\}}\right\rangle=$ $U^{\dagger}|k\rangle \otimes\left|\left\{n_{p}\right\}\right\rangle$. Consequently, $U$ provides a new point of view in which $\hat{H}$ does no longer describe independent excitations but refers to entangled exciton-phonon states. In this new point of view, a state $|k\rangle$ defines an exciton dressed by a virtual phonon cloud whereas the number state $\left|\left\{n_{p}\right\}\right\rangle$ describes phonons clothed by virtual excitonic transitions.

As shown in the next section, the new point of view is particularly suitable for deriving an approximate expression for the excitonic coherences. However, it is expected to give good results provided that the coupling between unperturbed states is smaller than the corresponding Bohr frequencies. By studying the different coupling paths, it turns out that the LFPM is responsible for the largest perturbation. Consequently, as shown previously $^{39}$, PT can be applied at temperature $T$ provided that ( $k_{B}$ is the Boltzmann constant)

$$
\frac{4 E_{B} k_{B} T}{\pi^{2} \Omega_{c}^{2}} L<x,
$$

where $x \approx 0.05$ specifies the desired accuracy for the approximate eigenenergies. For a given temperature and a fixed coupling strength, Eq.(8) shows that a critical length $L^{*}$ discriminates between two regimes. For $L<$ $L^{*}, \mathrm{PT}$ correctly describes the exciton-phonon dynamics whereas it certainly breaks down for $L>L^{*}$.

\section{Excitonic coherences}

Without any perturbation, the lattice is assumed to be in thermal equilibrium at temperature $T$. We thus consider situations for which $\omega_{0} \gg k_{B} T$ so that the exciton cannot be thermally excited. By contrast, the phonons form a thermal bath whose properties are described by the density matrix $\rho_{B}=\exp \left(-\beta H_{B}\right) / Z_{B}, Z_{B}$ being the phonon partition function $\left(\beta=1 / k_{B} T\right)$.

To study the exciton dynamics, the lattice is brought in a configuration out of equilibrium in which the exciton is prepared in a state $\left|\psi_{A}\right\rangle \neq|\oslash\rangle^{35-39}$. This step is supposed to be rather fast when compared with the typical time evolution of the phonons. As a result, the initial density matrix is defined as $\rho(0)=\left|\psi_{A}\right\rangle\left\langle\psi_{A}\right| \otimes \rho_{B}$. Of course, $\left|\psi_{A}\right\rangle$ depends on the properties under study. To analyze energy transport, one can choose $\left|\psi_{A}\right\rangle=\left|x_{0}\right\rangle$ indicating that an excitonic population is created on the $x_{0}$ th site ${ }^{46-50}$. In the present approach, we consider that $\left|\psi_{A}\right\rangle$ is a superimposition involving the vacuum $|\oslash\rangle$ and the one-exciton eigenstate $\left|k_{1}\right\rangle$ as $\left|\psi_{A}\right\rangle=c_{0}|\oslash\rangle+c_{1}\left|k_{1}\right\rangle$, with $\left|c_{0}\right|^{2}+\left|c_{1}\right|^{2}=1$. Note that such a superimposition may result from an optical excitation of the lattice.

In that context, the exciton properties are encoded in the $\operatorname{RDM} \sigma(t)=\operatorname{Tr}_{B}[\rho(t)]$, where $\operatorname{Tr}_{B}$ is a partial trace over the phonon degrees of freedom. Because $H$ conserves the exciton number, $\sigma(t)$ exhibits independent blocks. Matrix elements $\sigma_{x x}(t)$ and $\sigma_{x x^{\prime}}(t)$ define the one-exciton block that is required to study the transport properties ${ }^{48-50}$. Here, we restrict our attention to the block containing the coherences written as $\sigma_{k_{2} \oslash}(t)=G_{k_{2} k_{1}}(t) \sigma_{k_{1} \oslash}(0)\left(\sigma_{k_{1} \oslash}(0)=c_{0}^{*} c_{1}\right)$ with

$$
G_{k_{2} k_{1}}(t)=\left\langle k_{2}\left|\operatorname{Tr}_{B}\left[\rho_{B} e^{i H_{B} t} e^{-i H t}\right]\right| k_{1}\right\rangle .
$$

The coherence $\sigma_{k_{2} \oslash}(t)$ measures the ability of the exciton to develop a superimposition involving $|\oslash\rangle$ and $\left|k_{2}\right\rangle$ at time $t$, given that a superimposition involving $|\oslash\rangle$ and $\left|k_{1}\right\rangle$ was initially created. Consequently, $G_{k_{2} k_{1}}(t)$ generalizes the concept of transition amplitude and it defines the effective exciton propagator. It yields the probability amplitude to observe the exciton in $\left|k_{2}\right\rangle$ at time $t$ given that it was in $\left|k_{1}\right\rangle$ at $t=0$. More precisely, the full system being prepared in the factorized state $\left|k_{1}\right\rangle \otimes\left|\left\{n_{p}\right\}\right\rangle, G_{k_{2} k_{1}}(t)$ measures the probability 
amplitude to observe the system in a factorized state $\exp \left(-i \sum_{p} n_{p} \Omega_{p} t\right)\left|k_{2}\right\rangle \otimes\left|\left\{n_{p}\right\}\right\rangle$. It thus describes an excitonic transition during which the phonons evolve freely, an average over the initial phonon state being performed.

The evaluation of Eq.(9) within PT is detailed in Appendix B. To proceed, we first introduce $U$ and diagonalize $H$. Second, we use the fact that $\hat{H}$ is the sum of independent contributions, each contribution describing the exciton-phonon system when the exciton occupies a specific state $|k\rangle$. Then, we define the following Heisenberg representation $O_{k}(t)=e^{i \hat{H}_{B}^{(k)} t} O e^{-i \hat{H}_{B}^{(k)} t}$. Finally, we introduce the effective density matrix

$$
\rho_{B}^{(k)}(t)=\frac{1}{Z_{B}^{(k)}(t)} \prod_{p=1}^{N} \exp \left[-\left(\beta \Omega_{p}+i \delta \Omega_{p k} t\right) a_{p}^{\dagger} a_{p}\right]
$$

where

$$
Z_{B}^{(k)}(t)=\prod_{p=1}^{N} \frac{1}{1-\exp \left[-\left(\beta \Omega_{p}+i \delta \Omega_{p k} t\right)\right]} .
$$

Strictly speaking, $\rho_{B}^{(k)}(t)$ is not a density matrix since it provides complex values for the population of the phonon number states. However, it reduces to the equilibrium density matrix at $t=0$, i.e. $\rho_{B}^{(k)}(t=0) \equiv \rho_{B} \forall k$. Moreover, $\rho_{B}^{(k)}(t)$ being isomorphic to $\rho_{B}$, the trace over the phonon degrees of freedom will provide averages equivalent to thermal averages but with the correspondence $\beta \Omega_{p} \rightarrow \beta \Omega_{p}+i \delta \Omega_{p k} t$.

In that context, after simple algebraic manipulations, the effective propagator is rewritten as (see Appendix B)

$$
\begin{aligned}
G_{k_{2} k_{1}}(t)= & \sum_{k=1}^{N} \frac{Z_{B}^{(k)}(t)}{Z_{B}} \exp \left[-i\left(\omega_{k}+\delta \omega_{k}\right) t\right] \times \\
& \operatorname{Tr}_{B}\left[\rho_{B}^{(k)}(t)\left\langle k_{2}\left|U_{k}^{\dagger}(t)\right| k\right\rangle\left\langle k\left|U_{k}(0)\right| k_{1}\right\rangle\right]
\end{aligned}
$$

Expanding $U$ in a Taylor series with respect to $V$, one finally obtains the second order expression of the effective exciton propagator displayed in Appendix C (Eq.(C1)).

\section{NUMERICAL RESULTS}

In this section, the previous formalism is used for describing excitonic coherences in a finite size lattice. In spite of its general nature, it will be applied to amide-I exciton in $\alpha$-helices, a system extensively studied in our previous works ${ }^{35-39}$. In $\alpha$-helices, peptide units linked by $\mathrm{H}$ bonds are regularly distributed. Each unit contains an amide-I mode (high frequency $\mathrm{C}=\mathrm{O}$ vibration) that gives rise to vibrational excitons coupled with the phonons of the $\mathrm{H}$ bond network. This system is a prototype of a finite size lattice since helices containing from 3 to 15 residues are the most abundant in nature ${ }^{51}$. Of course, the present model is too simple to accurately describe vibrational energy flow in real helices whose dynamics exhibits a tremendous complexity due to the large number of degrees of freedom. Nevertheless, it involves ingredients that play a key role in interpreting specific experiments such as pump-probe spectroscopy in $\alpha$-helices ${ }^{13}$ and Electron Capture Dissociation in finite size polypeptides ${ }^{18}$. Unfortunately, this model is unable to describe energy relaxation because it conserves the number of amide-I exciton. In proteins, the amide-I lifetime, typically of about 1 ps, results from intramolecular energy redistribution due to the anharmonic coupling between each amide-I mode and a set of intramolecular normal modes whose displacements are strongly localized on the $\mathrm{C}=\mathrm{O}$ groups ${ }^{52-54}$. Consequently, we do not claim that the model is relevant to explain in details the vibrational dynamics in a protein. Nevertheless, its interest lies in the fact that it provides a simple approach to promote the idea that $\mathrm{PT}$ is more efficient than $\mathrm{TCL}_{2}$ method which has been applied recently to a similar model ${ }^{38}$.

Typical values for the parameters are used: $\omega_{0}=1660$ $\mathrm{cm}^{-1}, W=15 \mathrm{Nm}^{-1}, M=1.8 \times 10^{-25} \mathrm{~kg}, \Omega_{c}=96.86$ $\mathrm{cm}^{-1}$ and $\Phi=7.8 \mathrm{~cm}^{-1}$. The adiabaticity $B=2 \Phi / \Omega_{c}$ is equal to 0.16 so that the nonadiabatic limit is reached. The coupling strength will be discussed in terms of the $\chi$ parameter usually introduced in the Davydov model $\left(E_{B}=\chi^{2} / W\right)$. It will vary around $\chi=8 \mathrm{pN}$, a value close to ab initio estimate ${ }^{55}$. With these parameters, the critical length $L^{*} \approx 20$ ensures the PT validity over the temperature range $T \in[0,300] \mathrm{K}$ (see Eq.(8)).

As shown in Fig. 1a, the phonons induce a red-shift of each excitonic eigenenergy that scales as $\delta \omega_{k} \approx-E_{B}$. Nevertheless, $\delta \omega_{k}$ depends on both $k$ and $N$. A small dispersion arises and $\delta \omega_{k}$ increases with $k(\Phi>0)$. For $N=$ $10, \delta \omega_{1}=-0.99 E_{B}$ whereas $\delta \omega_{10}=-0.71 E_{B}$. Consequently, $\delta \omega_{k}$ defines a band centered on a mean value $\delta \bar{\omega}$ and whose width is about $\Delta \omega=\delta \omega_{N}-\delta \omega_{1}$. It turns out that $\delta \omega_{k}$ rapidly decreases with $L$ and it finally converges. Such a behavior is encoded in the size dependence of the band center that scales as $\delta \bar{\omega} \approx-1.056 E_{B}(1-1.96 / L)$. Nevertheless, since all the corrections behave similarly, the bandwidth is less sensitive to the lattice size. It scales as $\Delta \omega \approx\left(0.28-2.48 / L^{2.86}\right) E_{B}$ and it is about $0.28 E_{B}$ provided that $L>7$.

As displayed in Figs. 1b and 1c, the energy of each phonon mode is either red-shifted or blue-shifted due to the dressing by virtual excitonic transitions. In a general way, an exciton whose energy is above $\omega_{0}$ yields a red-shift of the phonon energy whereas an exciton whose energy is below $\omega_{0}$ favors a blue-shift. Nevertheless, opposite situations may appear, especially when the exciton occupies states close to the band center. For $N=8$, the exciton in state $k=4$ produces a blue-shift of the frequency of the phonon mode $p=5$ whereas the exciton in state $k=5$ yields a red-shift. In addition, specific phonon modes remain insensitive to the exciton that occupies a particular state. For $N=8$, when the exciton is either in the state $k=3$ or $k=6$, the phonon mode 

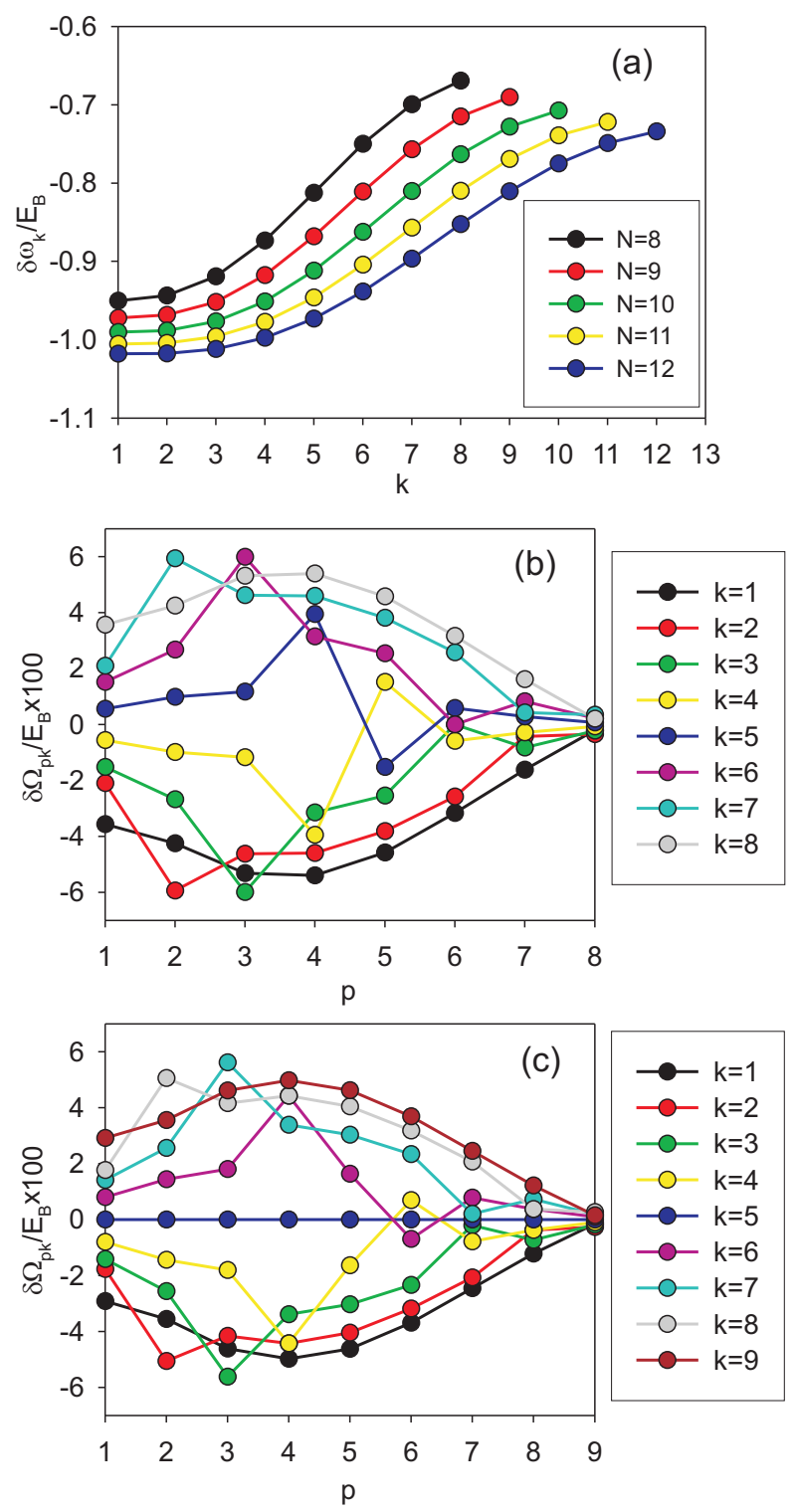

FIG. 1: (a) Size dependence of the exciton energy correction $\delta \omega_{k} / E_{B}$ vs $k$. (b) Phonon energy correction $\delta \Omega_{p k} / E_{B}$ vs $p$ for $N=8$ and for different $k$ values. (c) Phonon energy correction $\delta \Omega_{p k} / E_{B}$ vs $p$ for $N=9$ and for different $k$ values.

$p=6$ is not perturbed. As shown in Fig. 1c, a remarkable effect arises for odd $N$ values. In that case, phonons dressed by an exciton whose energy is exactly located at the band center are not perturbed. For $k=L / 2$, one obtains $\delta \Omega_{p k}=0 \forall p$. Note that the intensity of $\delta \Omega_{p k}$ decreases with the lattice size. Phonon modes with $p \approx L / 2$ exhibit the largest energy shift whereas phonon modes with $p \approx N$ seem to be less affected.

Let us now study the time evolution of the excitonic coherences. To proceed, we shall characterize the diagonal elements of the effective propagator, only. We have verified that such a restriction is valid because the propagator is diagonally dominant in the nonadiabatic weak coupling limit. Therefore, to simplify the notation, $G_{k k}(t)$ will be



FIG. 2: Time evolution of the coherence $\left|G_{k k}(t)\right|$ for $N=8$, $\chi=8 \mathrm{pN}$ and (a) $T=100 \mathrm{~K}$ and (b) $T=300 \mathrm{~K}$ (The inset illustrates the short time behavior for $T=100 \mathrm{~K})$.

called the coherence of the state $k$. For an even lattice size $(N=8)$, the time evolution of $\left|G_{k k}(t)\right|$ is illustrated in Fig. 2 a for $T=100 \mathrm{~K}$. Over a short time scale $(t<100$ ps), $\left|G_{k k}(t)\right|$ shows high frequency small amplitude fluctuations just below its initial value equal to unity (inset in Fig. 2a). Then, it follows a smooth decaying function that supports a high frequency small amplitude modulation indicating that quantum decoherence takes place. Such a behavior is strongly $k$ dependent. Indeed, the coherences evolve in pairs since $\left|G_{k k}(t)\right| \approx\left|G_{L-k L-k}(t)\right|$. Then, the coherence of states close to the band edges $\left(\omega_{k} \approx \omega_{0} \pm 2 \Phi\right)$ decays faster than the coherence of states located near the band center $\left(\omega_{k} \approx \omega_{0}\right)$. For instance, at $t=100 \mathrm{ps},\left|G_{11}(t)\right|=0.72$ and $\left|G_{44}(t)\right|=0.92$ whereas at $t=500 \mathrm{ps},\left|G_{11}(t)\right|=0.04$ and $\left|G_{44}(t)\right|=0.56$. Finally, the modulation amplitude is more pronounced for states located near the band center. As shown in Fig. 2b, the temperature enhances quantum decoherence. Consequently, $\left|G_{k k}(t)\right|$ remains close to its initial value over a shorter time scale of about 50 ps. Then, the larger $T$ is, the faster the coherences decay. Finally, as $T$ increases, the amplitude of the modulation increases. Nevertheless, the temperature affects differently the various coherences. At $t=200 \mathrm{ps},\left|G_{11}(t)\right|$ reduces from 0.37 for $T=100 \mathrm{~K}$ to 0.015 for $T=300 \mathrm{~K}$. By contrast, $\left|G_{44}(t)\right|$ varies between 0.86 and 0.43 over the same temperature range. Finally, over 1000 ps, Fig. 2b seems to indicate that the coherences vanish in the long time limit.

The time evolution of $\left|G_{k k}(t)\right|$ is shown in Fig. 3 for an odd lattice size $(N=9)$. Basically, the coherences 


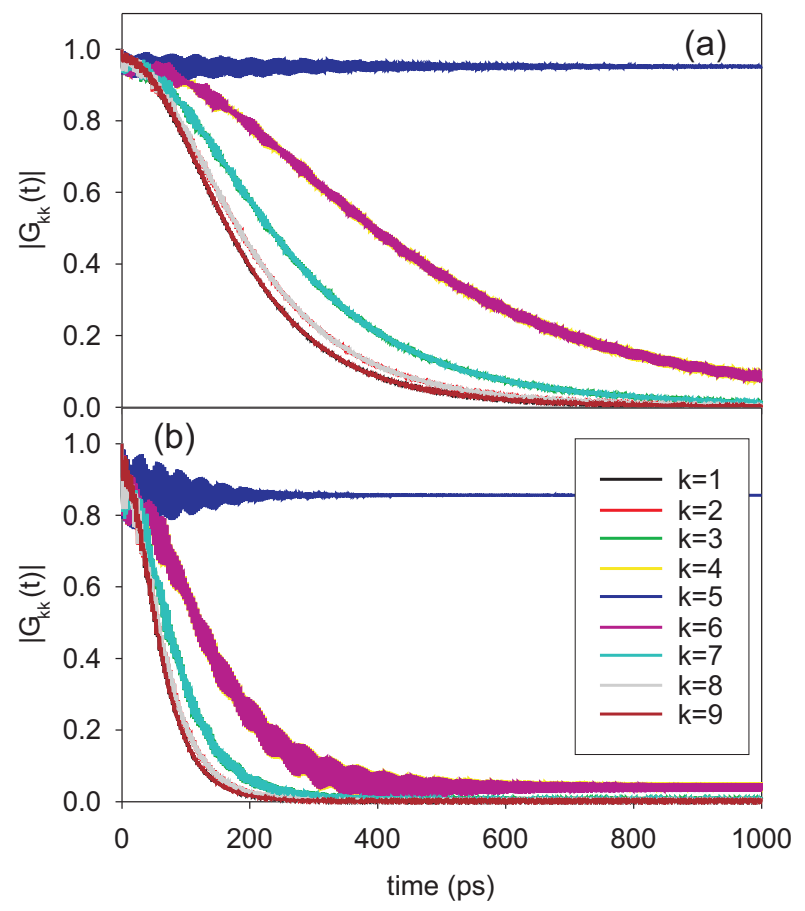

FIG. 3: Time evolution of the coherence $\left|G_{k k}(t)\right|$ for $N=9$, $\chi=8 \mathrm{pN}$ and (a) $T=100 \mathrm{~K}$ and (b) $T=300 \mathrm{~K}$.

behave as in Fig. 2. They still evolve in pairs and they first exhibit fast oscillations just below their initial value. As time increases, they follow smooth decaying functions that show high frequency small amplitude modulations. Quantum decoherence occurs and, as previously, it is enhanced by the temperature. Nevertheless, few differences take place. First, the modulation amplitude appears larger, especially for states located close to the band center. Then, the coherences that decay do no longer vanish in the long time limit. This is particularly pronounced at high temperature (Fig. 3b) for which the coherences converge to a finite value by exhibiting high frequency oscillations. The closer to the band center the state is located, the larger the asymptotic coherence value and the oscillation amplitude are. However, a surprising effect takes place that is absent for even $N$ values. Indeed, the coherence of the state exactly located at the band center $(k=L / 2)$ behaves differently. In the short time limit, it slightly decreases from unity by exhibiting high frequency damped oscillations. As time increases, the oscillations disappear and the coherence converges to a constant value $C_{L / 2}$ rather close to unity. The influence of the temperature is twofold. First, as $T$ increases, $C_{L / 2}$ decreases. Then, the amplitude of the damped oscillations increases with $T$. Nevertheless, the larger the temperature is, the faster the modulation disappears.

As illustrated in Fig. 4 for $N=8$ and $T=100 \mathrm{~K}$, quantum decoherence is not irreversible and specific features arise in the long time limit. Indeed, each coherence decreases until it recurs at specific revival times. For instance, although $\left|G_{11}(t)\right|$ almost vanishes after $1000 \mathrm{ps}$,

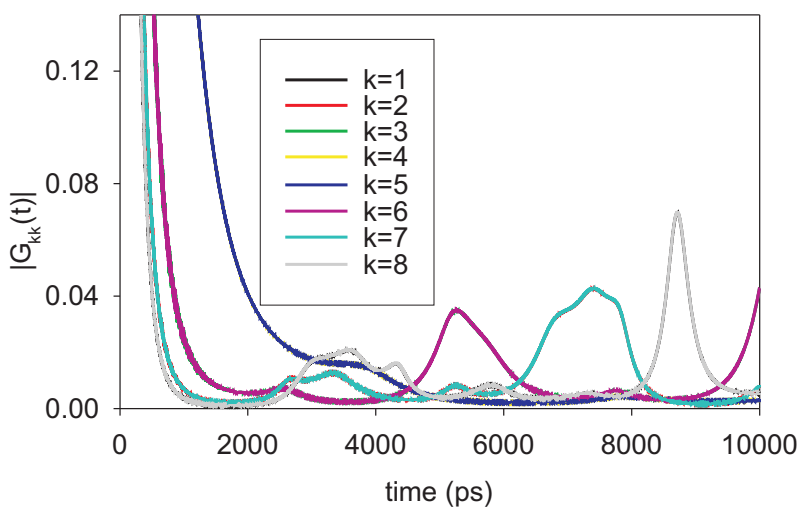

FIG. 4: Long time evolution of the coherence $\left|G_{k k}(t)\right|$ for $N=8, T=100 \mathrm{~K}$ and $\chi=8 \mathrm{pN}$.

it increases again over a time scale which extends from $2900 \mathrm{ps}$ to $4400 \mathrm{ps}$. Then additional recurrences occur around $5800 \mathrm{ps}, 7300 \mathrm{ps}$ and $8700 \mathrm{ps}$, the amplitude of which being smaller than 0.08 . However, we have verified that the recurrences decrease as $T$ increases so that they almost disappear at room temperature. For instance, for $N=8, T=300 \mathrm{~K}$ and $k=1$, recurrences still arise but their amplitudes remain smaller than 0.001. Similarly, the larger $N$ is, the smaller the recurrences are.

By contrast, even over very long time scales, the coherence of the state $k=L / 2$ survives and it rapidly converges to $C_{L / 2}$. As shown in Fig. $5 \mathrm{a}, C_{L / 2}$ decreases almost linearly as $T$ increases. A nonlinear dependence arises at very low temperature, only. For $N=9$ and $\chi=8 \mathrm{pN}, C_{L / 2}=0.95$ for $T=100 \mathrm{~K}$ whereas $C_{L / 2}=0.85$ for $T=300 \mathrm{~K}$. Moreover, the coupling enhances the temperature effect. Similarly, $C_{L / 2}$ is a linear function of the lattice size (Fig. 5b). For $T=300 \mathrm{~K}$ and $\chi=8 \mathrm{pN}$, it decreases from 0.92 to 0.71 when $N$ varies between 5 and 17. Finally, as shown in Fig. $5 \mathrm{c}, C_{L / 2}$ also decreases linearly with $E_{B}$ and it decays from 0.85 to 0.67 when $E_{B}$ varies between $0.21 \mathrm{~cm}^{-1}(\chi=8 \mathrm{pN})$ and $0.47 \mathrm{~cm}^{-1}(\chi=12 \mathrm{pN})$. These results reveal that the asymptotic value of the coherence of the state $k=L / 2$ scales as $C_{L / 2}=1-\alpha(N-1)$, with $\alpha \approx 3.82 E_{B} k_{B} T / \Omega_{c}^{2}$.

Let us finally characterize the decoherence rate $\Gamma_{k}$. It is defined as $\Gamma_{k}=1 / T_{k}$, where the decoherence time $T_{k}$ is extracted from the relation $\left|G_{k k}\left(T_{k}\right)\right|=1 / 2$. To proceed, we use the partition detailed in Appendix $\mathrm{C}$ and approximate $\left|G_{k k}(t)\right|$ by $\left|G_{k k}^{0}(t)\right|$, i.e. the expression of the coherences obtained when the system eigenstates are assumed to be collinear to the unperturbed states (see Eq.(C2)). We have verified that $\left|G_{k k}^{0}(t)\right|$ defines a smooth function that mimics the average behavior of the coherences. Its thus accounts for quantum decoherence so that both $T_{k}$ and $\Gamma_{k}$ can be extracted form the equation $\left|G_{k k}^{0}\left(T_{k}\right)\right|=\left|G_{k k}^{0}(0)\right| / 2$. As shown in Fig. 6a for $T=300 \mathrm{~K}$ and $\chi=8 \mathrm{pN}, \Gamma_{k}$ is a symmetric function with respect to the band center, i.e. $\Gamma_{k}=\Gamma_{L-k}$. It exhibits discrete values that belong to a band whose upper bound is $\Gamma_{1}$. The lower bound is either zero or $\Gamma_{N / 2}$, 

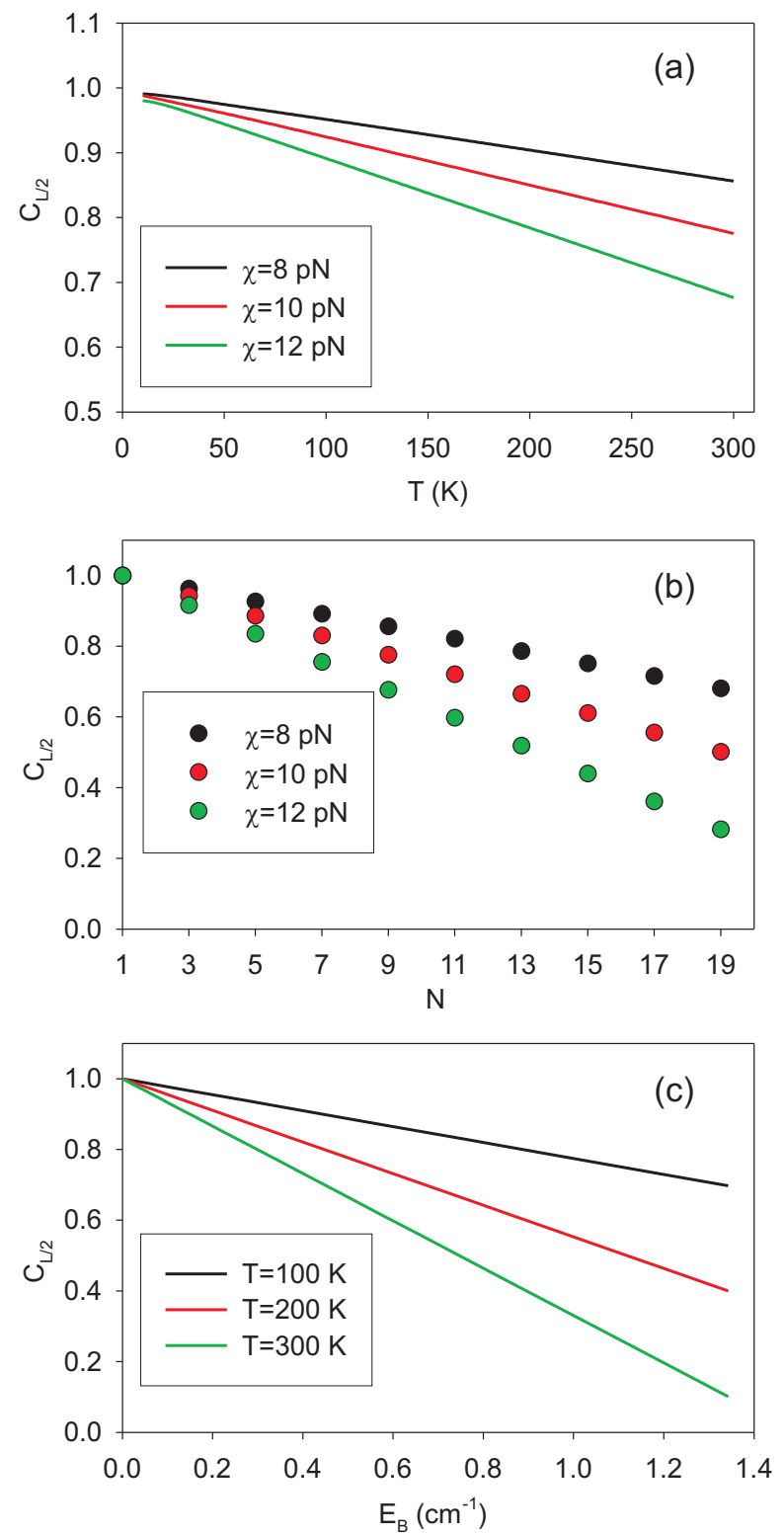

FIG. 5: Behavior of the asymptotic value $C_{L / 2}$ of the coherence of the state $k=L / 2$ versus (a) the temperature $(N=9)$, (b) the lattice size $(T=300 \mathrm{~K})$ and $(\mathrm{c})$ the coupling strength $(N=9)$

depending on the lattice size parity. For instance, for $N=8$, the decoherence rate extends from $\Gamma_{4}=0.028$ $\mathrm{cm}^{-1}$ to $\Gamma_{1}=0.099 \mathrm{~cm}^{-1}$. By contrast, for $N=9$, it ranges between $\Gamma_{5}=0$ and $\Gamma_{1}=0.096 \mathrm{~cm}^{-1}$. Note that we have verified that $\Gamma_{1}$ decreases with the lattice size and typically scales $1 / \sqrt{L}$. As illustrated in Fig. $6 \mathrm{~b}$ for $N=9$ and $\chi=8 \mathrm{pN}, \Gamma_{k}$ increases linearly with the temperature. Note that a nonlinear dependence arises at very low temperature, only. However, such a behavior strongly depends on the excitonic state. First of all, $\Gamma_{L / 2}=0 \forall T$. Then, the closer to the band edge the state is located, the faster $\Gamma_{k}$ increases with the temperature. For instance, $\Gamma_{1}$ varies between $0.031 \mathrm{~cm}^{-1}$ and
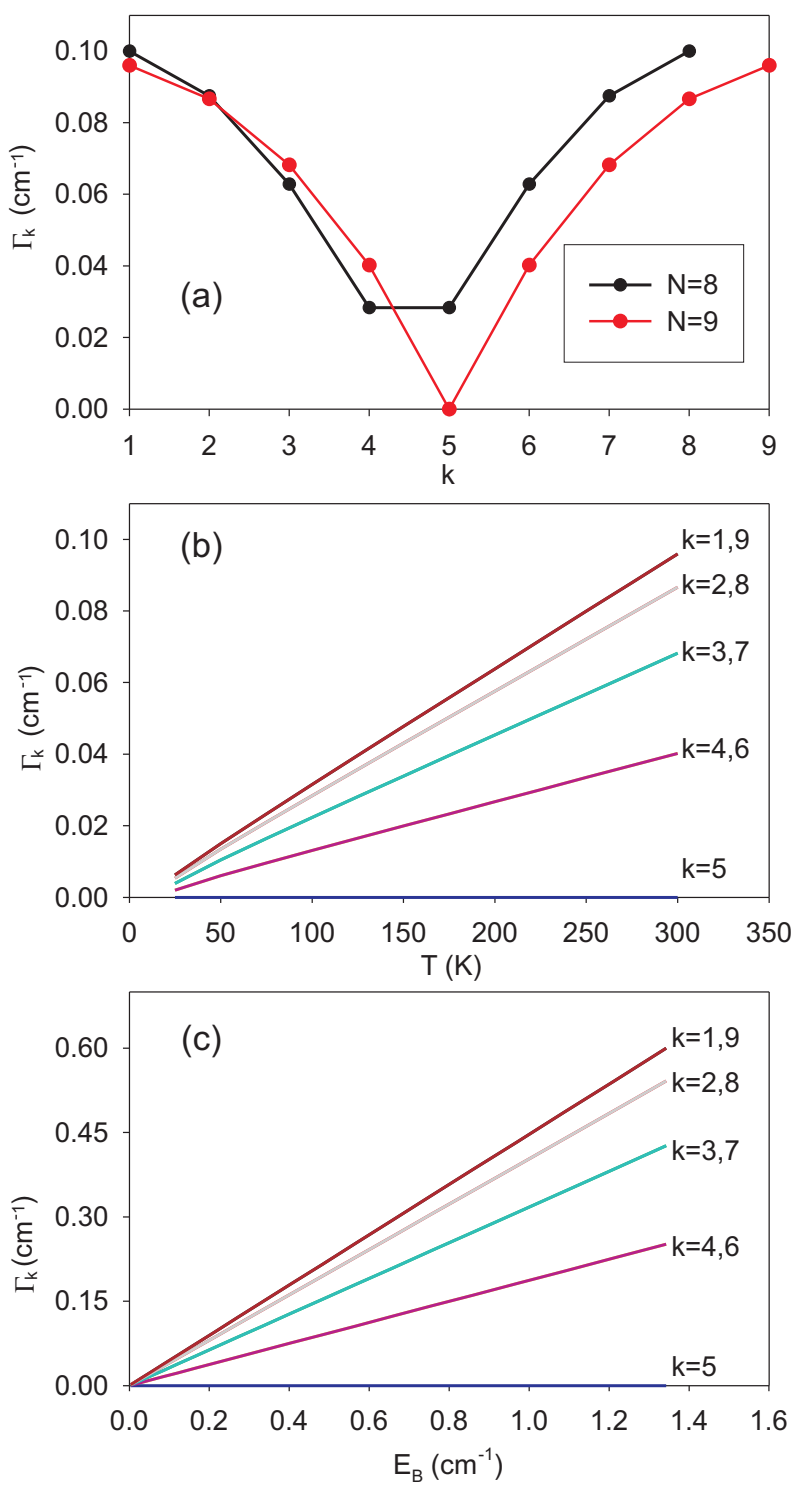

FIG. 6: Behavior of the decoherence rate $\Gamma_{k}$. (a) $\Gamma_{k}$ vs $k$ for $T=300 \mathrm{~K}, \chi=8 \mathrm{pN}$. (b) $\Gamma_{k}$ vs $T$ for $N=9$ and $\chi=8 \mathrm{pN}$. (c) $\Gamma_{k}$ vs $E_{B}$ for $N=9$ and $T=300 \mathrm{~K}$.

$0.096 \mathrm{~cm}^{-1}$ when $T$ increases from 100 to $300 \mathrm{~K}$. Over the same temperature range, $\Gamma_{4}$ extends from $0.013 \mathrm{~cm}^{-1}$ to $0.040 \mathrm{~cm}^{-1}$. In other words, for $T=100 \mathrm{~K}$, the decoherence time for the state $k=1$ is equal to $T_{1}=168.5 \mathrm{ps}$ whereas it reaches $T_{4}=405.5 \mathrm{ps}$ for the state $k=4$. For $T=300 \mathrm{~K}$, one obtains $T_{1}=55.5 \mathrm{ps}$ and $T_{4}=131.5 \mathrm{ps}$. Note that $T_{5}=\infty \forall T$. Similarly, $\Gamma_{k}$ increases linearly with $E_{B}$ in a way which depends on the $k$ values (Fig. $6 \mathrm{c})$. For $N=9$ and $T=300 \mathrm{~K}$, one typically obtains $\Gamma_{1} \approx 0.45 E_{B}$ and $\Gamma_{4} \approx 0.19 E_{B}$. Note that, as previously, $\Gamma_{L / 2}=0 \forall E_{B}$. 


\section{DISCUSSION}

In a confined environment, the coherence dynamics is controlled by different time scales. Over a short time scale, the coherences fluctuate just below their initial value equal to unity. Then, quantum decoherence takes place and each coherence follows a smooth decaying function that supports a small amplitude modulation. After a time scale specified by the decoherence time, the coherence either vanishes or exhibits small amplitude oscillations close to zero, depending on the parity of the lattice size. Finally, coherence revivals occur in the long time limit defining small recurrences whose amplitude decreases with both $T$ and $N$. The main observation is that quantum decoherence strongly depends on the exciton eigenstate. The closer to the band center the state is located, the longer the decoherence time is. In particular, for odd lattice sizes, the coherence of the state exactly located at the band center survives over an infinite time scale, even at room temperature.

To interpret these features, let us first mention that within PT, the influence of the exciton-phonon interaction is encoded into two main parameters. The first parameter, $\delta \omega_{k}$, describes the energy shift experienced by the exciton when it is dressed by a virtual phonon cloud. According to the small polaron concept ${ }^{41,42}$, this dressing yields a red-shift of the Bohr frequency of each two-level system. This shift, equal to $E_{B}$ in an infinite lattice, reduced to $E_{B}(1-2 / L)$ in a confined environment ${ }^{42}$. Moreover, the dressed exciton delocalizes according to a reduced hopping constant $\hat{\Phi} \approx \Phi \exp (-S)$, where the band-narrowing factor at zero temperature is $S=8 E_{B} /\left(3 \pi \Omega_{c}\right)^{41}$. Note that in a finite size lattice $\hat{\Phi}$ is both inhomogeneous and size dependent ${ }^{56}$. But these effects remain weak in a lattice with fixed boundary conditions. As a result, the energy corrections behave as

$$
\delta \omega_{k} \approx-E_{B}(1-2 / L)-2 \Phi S \cos (k \pi / L) .
$$

As observed in Fig. 1, $\delta \omega_{k}$ forms a band centered on $\delta \bar{\omega}=-E_{B}(1-2 / L)$ and whose width $\Delta \omega=4 \Phi S$ reduces to $0.273 E_{B}$ with the parameters used in the figure.

The second parameter accounts for the fact that, dressed by the virtual transitions of an exciton in state $k$, the energy of a phonon $p$ becomes $\hat{\Omega}_{p}=\Omega_{p}+\delta \Omega_{p k}$. To evaluate the energy correction $\delta \Omega_{p k}$ displayed in Figs. 1b and 1c, let us approximate Eq.(7) by considering normal scattering processes $k^{\prime}=k-p$ and $k^{\prime}=k+p$, only. Up to first order in the adiabaticity, one obtains

$$
\delta \Omega_{p k} \approx-\frac{8 B E_{B}}{L}\left(\frac{\Omega_{p}}{\Omega_{c}}\right)\left[1-\left(\frac{\Omega_{p}}{\Omega_{c}}\right)^{2}\right] \cos \left(\frac{k \pi}{L}\right) .
$$

Eq.(14) shows that $\delta \Omega_{p k}<0$ if $k<L / 2$ whereas $\delta \Omega_{p k}>$ 0 if $k>L / 2$. It reveals that $\delta \Omega_{p k}=0 \forall p$ for $k=$ $L / 2$. The most perturbed phonon mode satisfying $\Omega_{p}=$ $\Omega_{c} / \sqrt{3}$, the maximum energy shift is $3.08 B E_{B} / L$, i.e. about $5 E_{B} / 100$ for $L=9-10$, as observed in Fig. 1 .
In that context, assuming that the system eigenstates reduce to the unperturbed states, the effective exciton propagator is rewritten as (see Eq.(C2) with $\Lambda_{k}^{(2)}=0$ ) $G_{k k}(t) \approx \exp \left(-i \hat{\omega}_{k} t\right) Z_{B}^{(k)}(t) / Z_{B}$. The corresponding modulus is thus defined as

$$
\left|G_{k k}(t)\right| \approx \prod_{p=1}^{N} \frac{1}{\sqrt{1+4 \Delta \bar{n}_{p}^{2} \sin ^{2}\left(\delta \Omega_{p k} t / 2\right)}},
$$

where $\Delta \bar{n}_{p}^{2}=\bar{n}_{p}\left(\bar{n}_{p}+1\right)$ measures the thermal fluctuations of the $p$ th phonon number around its average value $\bar{n}_{p}=\left[\exp \left(\beta \Omega_{p}\right)-1\right]^{-1}$. Although it is quite simple, Eq.(15) provides a clear understanding of the different features observed in the previous section. First of all, since $\delta \Omega_{p L-k}=-\delta \Omega_{p k}$ (see Eq.(7)), it turns out that $\left|G_{k k}(t)\right|=\left|G_{L-k L-k}(t)\right|$, as observed in Figs. 2, 3 and 4 .

Then, Eq.(15) describes a smooth decaying function that tends to zero over intermediate time scale, revealing that quantum decoherence occurs. The physics of the decoherence can be understood as follows. At $t=0$, the system is prepared in a factorized state $|\Psi(0)\rangle=$ $\left[c_{0}|\oslash\rangle+c_{1}|k\rangle\right] \otimes|\phi\rangle$. This state describes an exciton in a superimposed state accompanied by phonons in a well defined number state $|\phi\rangle \equiv\left|\left\{n_{p}\right\}\right\rangle$, phonons and exciton being independent. As time increases, this state evolves in an entangled exciton-phonon state as

$$
|\Psi(t)\rangle \approx c_{0}|\oslash\rangle \otimes\left|\phi_{\oslash}(t)\right\rangle+c_{1} e^{-i \hat{\omega}_{k} t}|k\rangle \otimes\left|\phi_{k}(t)\right\rangle,
$$

where $\left|\phi_{\oslash}(t)\right\rangle$ is the state of free phonons whereas $\left|\phi_{k}(t)\right\rangle$ describes the state reached by dressed phonons. Building the system density matrix and performing a trace over the phonon degrees of freedom, one obtains $G_{k k}(t) \approx$ $\exp \left(-i \hat{\omega}_{k} t\right)\left\langle\phi_{\oslash}(t) \mid \phi_{k}(t)\right\rangle$. The phase factor, that does not affect the coherence dynamics, accounts for the evolution of the dressed exciton. By contrast, the inner product $\left\langle\phi_{\oslash}(t) \mid \phi_{k}(t)\right\rangle$ defines the so-called decoherence function that measures the ability of the phonons to evolve freely in spite of their coupling with the exciton. This object is rather general in the theory of open quantum systems ${ }^{28,29}$. It suggests that quantum decoherence results from the fact that the bath evolves differently as the open system occupies different quantum states (see for instance Ref. ${ }^{57}$ ). In the present situation, a slightly different interpretation emerges. Indeed, when the phonons are initially in a pure state, the inner product reduces to a phase factor $\prod_{p} \exp \left(i n_{p}\left(\Omega_{p}-\hat{\Omega}_{p k}\right) t\right)$ that involves the energy difference between free and dressed phonons. Although this phase factor accounts for the fact that dressed phonons evolve differently when compared with bare phonons, it does not affect the excitonic coherence. However, at finite temperature, the phonons are described by a statistical mixture of number states so that an average procedure is required. Therefore, performing the average over the initial phonon state yields a sum over phase factors which interfere some with the others, resulting in the decay of the excitonic coherence.

As shown in Eq.(15), describing the coherence decay remains a hard task since $\left|G_{k k}(t)\right|$ involves the product of 
periodic functions. To overcome this difficulty, we first express the coherence as $\left|G_{k k}(t)\right|=\exp \left[-F_{k}(t)\right], F_{k}(t)$ being the decoherence factor. Expanding $F_{k}(t)$ in the short time limit, one finally obtains

$$
\left|G_{k k}(t)\right| \approx \exp \left[-\frac{1}{2} \sum_{p=1}^{N} \Delta \bar{n}_{p}^{2} \delta \Omega_{p k}^{2} t^{2}\right] .
$$

Eq.(17) indicates that the coherence behaves as a Gaussian function whose half width at half maximum yields an approximate expression for the decoherence rate $\Gamma_{k}^{g}$. Note that a similar time evolution has been proposed to characterize quantum decoherence in a solute/bath system $^{57}$. However, we have verified that $\left|G_{k k}(t)\right|$ scales as a Gaussian function provided that $t<1 / \Gamma_{g}$. Over longer time scale, a slower decay takes place. Consequently, $\Gamma_{k}^{g}$ always over estimates the exact decoherence rate displayed in Fig. 6. However, after a detailed analysis of our numerical data, it turns out that the ratio $\Gamma_{k} / \Gamma_{k}^{g}$ only depends on the lattice size. Therefore, a quite good estimate of the decoherence rate that clearly reproduces the data displayed in Fig. 6 is expressed as

$$
\Gamma_{k} \approx \frac{1-1 / L}{\sqrt{2 \ln 2}} \sqrt{\sum_{p=1}^{N} \Delta \bar{n}_{p}^{2} \delta \Omega_{p k}^{2}} .
$$

As shown in Eq.(18), the temperature dependence of $\Gamma_{k}$ is encoded in the fluctuations of the phonon numbers. Since $\Delta \bar{n}_{p}$ typically scales as $k_{B} T / \Omega_{p}, \Gamma_{k}$ increases linearly with the temperature. Note that this dependence differs from the standard expression of the dephasing rate that characterizes the decoherence of an open system coupled with a reservoir of harmonic oscillators ${ }^{1}$. In that case, GME methods show that the temperature dependence of the rate originates in its dependence with respect to the average phonon number $\bar{n}_{p}$. At high temperature, both approaches yield a similar temperature dependence since $\Delta \bar{n}_{p} \approx \bar{n}_{p} \approx k_{B} T / \Omega$. This is no longer the case at low temperature since $\Delta \bar{n}_{p} / \bar{n}_{p}=\exp \left(\beta \Omega_{p} / 2\right)$. Moreover, $\Gamma_{k}$ is proportional to $E_{B}$ through its dependence with respect to the phonon energy corrections (see Eq.(7)). From this dependence, we recover that the closer to the band edges the excitonic state is located, the larger $\Gamma_{k}$ is.

As observed in Fig. 3, Eq.(18) also reveals that the coherence of the state located at the band center $(k=L / 2)$ survives over an infinite time scale. This feature occurs for an odd lattice size only because $\delta \Omega_{p L / 2}=0$ $\forall p$. Indeed, during both phonon absorption and phonon stimulated emission, the virtual excitonic transition form $|k\rangle$ to $\left|k^{\prime}\right\rangle$ is counterbalanced by the transition form $|k\rangle$ to $\left|L-k^{\prime}\right\rangle$. Due to the symmetry of the excitonic energy spectrum $\left(\left(\omega_{k}^{\prime}-\omega_{0}\right)=-\left(\omega_{L-k^{\prime}}-\omega_{0}\right)\right)$ and because $M_{p k k^{\prime}}=M_{p k L-k^{\prime}}$, both contributions exactly compensate when $k=L / 2$. As a result, a zero phonon energy shift occurs so that $\Gamma_{L / 2} \rightarrow 0$. Note that the asymptotic behavior of the coherence $C_{L / 2}$ is not described by
Eq.(15). As shown in Fig. 5, $C_{L / 2}$ decreases linearly with both $T, E_{B}$ and $N$. Such a behavior originates in the second order correction of the eigenstates that ensure their normalization to unity. As shown in Eq.(C2), this correction yields the contribution $\Lambda_{k}^{(2)}(t)$ which provides to $C_{L / 2}$ its dependence with respect to the model parameters.

Finally, Eq.(15) gives formation about the long time behavior of the coherences. Indeed, when restricting the influence of the phonon bath to the $p$ th mode, only, $\left|G_{k k}(t)\right|$ reduces to a periodic function of time with pe$\operatorname{riod} \tau_{p k}=2 \pi /\left|\delta \Omega_{p k}\right|$. As a result, although the coherence decays due to quantum decoherence, it finally recurs periodically at specific revivals time $\tau_{p k}, 2 \tau_{p k}, 3 \tau_{p k} \ldots$ and so on. These revivals time define exact recurrences for which the coherence reaches unity. However, this ideal scenario breaks down when all the phonon modes are considered. The main reason it that it is unlikely that the contribution of each phonon mode will recur simultaneously to provide a strong recurrence. In other words, it is as if the coherence revivals due to the coupling with a particular phonon mode were screened by the remaining modes. Nevertheless, each phonon mode yields specific features that can be distinguished in the long time behavior of the coherence at low temperature and in short lattices. For instance, with the parameters used in Fig. 4 , the coherence of the state $k=1$ slightly recurs over a time scale which extends from 2900 ps to 4400 ps. This feature is the signature of coherence revivals that take place at the revival times $\tau_{11}=4357 \mathrm{ps}, \tau_{21}=3655 \mathrm{ps}$, $\tau_{31}=2919 \mathrm{ps}, \tau_{41}=2873 \mathrm{ps}$ and $\tau_{51}=3398 \mathrm{ps}$. Moreover, the observed recurrences around $5800 \mathrm{ps}, 7300 \mathrm{ps}$ and $8700 \mathrm{ps}$ are located at the revival times $2 \tau_{31}, 2 \tau_{21}$ and $2 \tau_{11}$, respectively.

To conclude, let us discuss the implications of the present work for quantum information processing in condensed matter. Indeed, quantum state transfer (QST) from one region to another is a fundamental task in quantum computing ${ }^{58}$. Over short length scale, to ensure communication inside a computer or between adjacent computers, solid-state based system is the ideal candidate for scalable quantum computing. However, the quantum channel strongly depends on the way the information is encoded. Since it has been suggested that qubits may be encoded in high frequency vibrational modes ${ }^{59-61}$, vibrational exciton-mediated QST is a promising way for quantum information processing ${ }^{26,27}$.

In that context, the confinement of the excitons appears as a key ingredient to fight against quantum decoherence, the public enemy number one in quantum computing. In an infinite lattice, the exciton eigenstates are Bloch states whose coherent nature rapidly disappears due to the coupling with the phonon bath ${ }^{36}$. The coherences decay exponentially according to a state independent decoherence rate of about $\Gamma=4 E_{B} k_{B} T / \Omega_{c}$. With the parameters used in the present study, the corresponding decoherence time is about 2.86 ps at room temperature. By contrast, the confinement softens the decoher- 
ence experienced by the exciton, even at high temperature. Although quantum decoherence strongly depends on the excitonic states, the decoherence times typically range between 50 - 200 ps at $300 \mathrm{~K}$. Furthermore, it turns out that the excitonic state exactly located at the band center is insensitive to the phonon bath, its decoherence time being infinite. Of course, such a behavior results from a second order PT and high order theory will provide non vanishing $\Gamma_{L / 2}$ values. Nevertheless, even if quantum decoherence affects this state, one may expect that the decoherence time will be extremely long in the nonadiabatic weak coupling limit. A superimposition involving the vacuum and this particular one-exciton state will be able to keep its coherent nature over an extremely long time scale. At high temperature, such a superimposition can be viewed as an ideal qubit insensitive to quantum decoherence.

\section{CONCLUSION}

In this paper, the properties of a confined exciton coupled with phonons in thermal equilibrium has been revisited using the operatorial formulation of PT. This method provides a new point of view in which the dynamics is governed by an effective Hamiltonian that does no longer characterize independent excitations but accounts for exciton-phonon entanglement. The exciton is dressed by a virtual phonon cloud whereas the phonons are clothed by virtual excitonic transitions. Within this new point of view, special attention has been paid for describing the excitonic coherences that measure the ability of the exciton to develop superimpositions involving the vacuum and one-exciton states.

In the nonadiabatic weak coupling limit, it has been shown that the coherence behavior results from an average procedure over the phonon degrees of freedom. Indeed, when the phonons occupy a well-defined number state, the decoherence function reduces to a phase factor although dressed phonons behave differently when compared with bare phonons. At finite temperature, the average over the initial phonon state yields a sum over phase factors which interfere some with the others, resulting in the decay of the excitonic coherence. Therefore, although each coherence remains close to unity over a rather short time scale, it finally decreases as time increases. After a time scale specified by the decoherence time, the coherence either vanishes or shows small amplitude oscillations close to zero, depending on the parity of the lattice size. Coherence revivals have been observed in the long time limit, but their amplitude remains extremely small.

The key point is that the confinement softens quantum decoherence. Indeed, it has been shown that the coherences are very sensitive to the excitonic states and to the lattice size. Therefore, the closer to the band center the state is located, the slower the coherence decays. In particular, for odd lattice sizes, the coherence of the state exactly located at the band center survives over an infinite time scale. In that context, it has been pointed out that a superimposition involving the vacuum and this particular one-exciton state behaves as an ideal qubit insensitive to the phonon bath, even at room temperature.

To conclude, let us mention that $\mathrm{PT}$ is valid as long as we remain confined in a small region of the parameter space, i.e. in the weak coupling limit and for short lattice sizes. Therefore, a more general framework is required to correctly describe size effects in both intermediate and strong coupling limits. Based on the small polaron concept, these features will be addressed in forthcoming works.

\section{APPENDIX A: SECOND ORDER PERTURBATION THEORY}

In its operatorial formulation ${ }^{45}$, PT is based on the introduction of a unitary transformation $U$ that diagonalizes the transformed Hamiltonian $\hat{H}=U H U^{\dagger}$ in the unperturbed basis. It is written as $U=\exp (S)$, where $S$ is an anti-hermitian operator assumed to be non-diagonal in the unperturbed basis. It is expanded as a Taylor series as $S=S_{1}+S_{2}+S_{3}+\ldots$ where $S_{q}$ is the $q$ th order correction in the coupling $V$. Consequently, $\hat{H}$ becomes

$$
\begin{aligned}
\hat{H} & =H_{0}+V+\left[S_{1}, H_{0}\right] \\
& +\left[S_{1}, V\right]+\left[S_{2}, H_{0}\right]+\frac{1}{2}\left[S_{1},\left[S_{1}, H_{0}\right]\right]+\ldots
\end{aligned}
$$

From Eq.(A1), $S$ is derived order by order to diagonalize $\hat{H}$ at the desired order. Up to second order in $V$, the solution of the problem is given by the following equations

$$
\begin{aligned}
& {\left[H_{0}, S_{1}\right]=V} \\
& {\left[H_{0}, S_{2}\right]=\frac{1}{2}\left[S_{1}, V\right]^{n d}} \\
& \hat{H}=H_{0}+\frac{1}{2}\left[S_{1}, V\right]^{d},
\end{aligned}
$$

where $d$ and $n d$ define the diagonal part and the nondiagonal part of an operator in the unperturbed basis.

Since $V$ is a linear combination of creation and annihilation phonon operators (see Eq.(2)), $S_{1}$ is of the form

$$
S_{1}=\sum_{p} \Lambda_{p} a_{p}^{\dagger}-\Lambda_{p}^{\dagger} a_{p} .
$$

The unknown operator $\Lambda_{p}$ acts in $\mathcal{E}_{A}$, only. No restriction affects this operator since $S_{1}^{\dagger}=-S_{1}$. Therefore, inserting Eq.(A3) into Eq.(A2) yields

$$
\left\langle k\left|\Lambda_{p}\right| k^{\prime}\right\rangle=\frac{\left\langle k\left|M_{p}\right| k^{\prime}\right\rangle}{\omega_{k}-\omega_{k^{\prime}}+\Omega_{p}} .
$$

The knowledge of $S_{1}$ allows us to compute the commutator $\left[S_{1}, V\right]$ that is required to derive both $\hat{H}$ and $S_{2}$. 
This commutator is defined as

$$
\begin{aligned}
\frac{1}{2}\left[S_{1}, V\right] & =\sum_{p} A_{p}+\sum_{p p^{\prime}} B_{p p^{\prime}} a_{p^{\prime}}^{\dagger} a_{p}^{\dagger}+B_{p p^{\prime}}^{\dagger} a_{p} a_{p^{\prime}} \\
& +\sum_{p p^{\prime}} B_{p p^{\prime}} a_{p}^{\dagger} a_{p^{\prime}}+B_{p p^{\prime}}^{\dagger} a_{p^{\prime}}^{\dagger} a_{p}
\end{aligned}
$$

where

$$
\begin{aligned}
B_{p p^{\prime}} & =\frac{1}{2}\left[\Lambda_{p}, M_{p^{\prime}}\right] \\
A_{p} & =-\frac{1}{2}\left(\Lambda_{p}^{\dagger} M_{p}+M_{p} \Lambda_{p}\right) .
\end{aligned}
$$

From the diagonal part of Eq.(A5), the transformed Hamiltonian $\hat{H}$ reduces to

$$
\hat{H}=H_{A}+\sum_{p} A_{p}^{d}+\sum_{p}\left[\Omega_{p}+2 B_{p p}^{d}\right] a_{p}^{\dagger} a_{p} .
$$

Within the excitonic eigenbasis, Eq.(A7) yields Eqs.(5)(7) with the definitions

$$
\begin{aligned}
\delta \omega_{k} & =\sum_{p=1}^{N}\left\langle k\left|A_{p}^{d}\right| k\right\rangle \\
\delta \Omega_{p k} & =2\left\langle k\left|B_{p p}^{d}\right| k\right\rangle .
\end{aligned}
$$

From the non diagonal part of Eq.(A5), one seeks $S_{2}$ of the form

$$
\begin{aligned}
S_{2} & =\sum_{p p^{\prime}} E_{p p^{\prime}} a_{p^{\prime}}^{\dagger} a_{p}^{\dagger}-E_{p p^{\prime}}^{\dagger} a_{p} a_{p^{\prime}} \\
& +\sum_{p p^{\prime}} D_{p p^{\prime}} a_{p}^{\dagger} a_{p^{\prime}}-D_{p p^{\prime}}^{\dagger} a_{p^{\prime}}^{\dagger} a_{p}+\sum_{p} C_{p},
\end{aligned}
$$

where the unknown operators $E_{p p^{\prime}}, D_{p p^{\prime}}$ and $C_{p}$ act in $\mathcal{E}_{A}$, only. Note that $C_{p}^{\dagger}=-C_{p}$ to ensure the anti-hermitian nature of $S_{2}$. By inserting Eq.(A9) into Eq.(A2), these operators are finally defined as

$$
\begin{aligned}
\left\langle k\left|C_{p}\right| k^{\prime}\right\rangle & =\frac{\left\langle k\left|A_{p}^{n d}\right| k^{\prime}\right\rangle}{\omega_{k}-\omega_{k^{\prime}}} \\
\left\langle k\left|D_{p p^{\prime}}\right| k^{\prime}\right\rangle & =\frac{\left\langle k\left|\bar{B}_{p p^{\prime}}\right| k^{\prime}\right\rangle}{\omega_{k}-\omega_{k^{\prime}}+\Omega_{p}-\Omega_{p^{\prime}}} \\
\left\langle k\left|E_{p p^{\prime}}\right| k^{\prime}\right\rangle & =\frac{\left\langle k\left|B_{p p^{\prime}}\right| k^{\prime}\right\rangle}{\omega_{k}-\omega_{k^{\prime}}+\Omega_{p}+\Omega_{p^{\prime}}},
\end{aligned}
$$

where $\bar{B}_{p p^{\prime}}=B_{p p}^{n d} \delta_{p p^{\prime}}+B_{p p^{\prime}}\left(1-\delta_{p p^{\prime}}\right)$.

\section{APPENDIX B: GENERAL EXPRESSION OF THE EXCITONIC COHERENCES}

By inserting the unitary transformation $U$, the coherence Eq.(9) is rewritten as

$$
G_{k_{2} k_{1}}(t)=\left\langle k_{2}\left|\operatorname{Tr}_{B}\left[\rho_{B} e^{i H_{B} t} U^{\dagger} e^{-i \hat{H} t} U\right]\right| k_{1}\right\rangle .
$$

Since $\hat{H}$ is a sum of independent contribution (see Eq.(5)), the coherence is expressed as

$$
\begin{aligned}
G_{k_{2} k_{1}}(t)= & \sum_{k=1}^{N} \exp \left[-i\left(\omega_{k}+\delta \omega_{k}\right) t\right] \times \\
& \operatorname{Tr}_{B}\left[\rho_{B} e^{i H_{B} t}\left\langle k_{2}\left|U^{\dagger}\right| k\right\rangle e^{-i \hat{H}_{B}^{(k)} t}\left\langle k|U| k_{1}\right\rangle\right] .
\end{aligned}
$$

Because the operator $\exp \left(-i \hat{H}_{B}^{(k)} t\right)$ defines a unitary evolution and since $\left[H_{B}, \hat{H}_{B}^{(k)}\right]=0$, the partial trace in Eq.(B2) is rewritten as

$$
\operatorname{Tr}_{B}\left[\rho_{B} e^{i\left(H_{B}-\hat{H}_{B}^{(k)}\right) t}\left\langle k_{2}\left|e^{i \hat{H}_{B}^{(k)} t} U^{\dagger} e^{-i \hat{H}_{B}^{(k)} t}\right| k\right\rangle\left\langle k|U| k_{1}\right\rangle\right]
$$

At this step, let first define $U_{k}(t)=e^{i \hat{H}_{B}^{(k)}} t U e^{-i \hat{H}_{B}^{(k)} t}$ as the Heisenberg representation of the unitary transformation $U$ with respect to the Hamiltonian $\hat{H}_{B}^{(k)}$. Then, since $\rho_{B}=\exp \left(-\beta H_{B}\right) / Z_{B}$, one obtains

$$
\rho_{B} e^{i\left(H_{B}-\hat{H}_{B}^{(k)}\right) t}=\frac{Z_{B}^{(k)}(t)}{Z_{B}} \rho_{B}^{(k)}(t),
$$

with

$$
\begin{aligned}
& \rho_{B}^{(k)}(t)=e^{-\beta H_{B}+i t\left(H_{B}-\hat{H}_{B}^{(k)}\right)} / Z_{B}^{(k)}(t) \\
& Z_{B}^{(k)}(t)=\operatorname{Tr}_{B}\left[e^{-\beta H_{B}+i t\left(H_{B}-\hat{H}_{B}^{(k)}\right)}\right] .
\end{aligned}
$$

From the expressions of both $H_{B}$ and $H_{B}^{(k)}$, Eq.(B3) can be easily evaluated to finally obtain Eqs.(10) and (11). Therefore, inserting Eq.(B3) into the previous expression of the partial trace and combining the results with Eq.(B2) yield the effective propagator Eq.(12).

\section{APPENDIX C: SECOND ORDER EXPRESSION OF THE EXCITONIC COHERENCES}

The approximate expression of the effective exciton propagator up to second order in the exciton-phonon coupling $V$ is written as 


$$
\begin{aligned}
G_{k_{2} k_{1}}(t) & =\frac{Z_{B}^{\left(k_{1}\right)}(t)}{Z_{B}} e^{-i \hat{\omega}_{k_{1}} t} \delta_{k_{2}, k_{1}} \\
& -\frac{Z_{B}^{\left(k_{2}\right)}(t)}{2 Z_{B}} e^{-i \hat{\omega}_{k_{2}} t} \sum_{p}\left[\left\langle k_{2}\left|\Lambda_{p} \Lambda_{p}^{\dagger}\right| k_{1}\right\rangle n_{p}^{\left(k_{2}\right)}(t)+\left\langle k_{2}\left|\Lambda_{p}^{\dagger} \Lambda_{p}\right| k_{1}\right\rangle\left(n_{p}^{\left(k_{2}\right)}(t)+1\right)\right] \\
& -\frac{Z_{B}^{\left(k_{1}\right)}(t)}{2 Z_{B}} e^{-i \hat{\omega}_{k_{1}} t} \sum_{p}\left[\left\langle k_{2}\left|\Lambda_{p} \Lambda_{p}^{\dagger}\right| k_{1}\right\rangle n_{p}^{\left(k_{1}\right)}(t)+\left\langle k_{2}\left|\Lambda_{p}^{\dagger} \Lambda_{p}\right| k_{1}\right\rangle\left(n_{p}^{\left(k_{1}\right)}(t)+1\right)\right] \\
& +\frac{Z_{B}^{\left(k_{2}\right)}(t)}{Z_{B}} e^{-i \hat{\omega}_{k_{2}} t} \sum_{p}\left[\left\langle k_{2}\left|C_{p}\right| k_{1}\right\rangle+\left\langle k_{2}\left|D_{p p}-D_{p p}^{\dagger}\right| k_{1}\right\rangle n_{p}^{\left(k_{2}\right)}(t)\right] \\
& -\frac{Z_{B}^{\left(k_{1}\right)}(t)}{Z_{B}} e^{-i \hat{\omega}_{k_{1}} t} \sum_{p}\left[\left\langle k_{2}\left|C_{p}\right| k_{1}\right\rangle+\left\langle k_{2}\left|D_{p p}-D_{p p}^{\dagger}\right| k_{1}\right\rangle n_{p}^{\left(k_{1}\right)}(t)\right] \\
& +\sum_{k} \frac{Z_{B}^{(k)}(t)}{Z_{B}} e^{-i \hat{\omega}_{k} t} \sum_{p} e^{i\left(\Omega_{p}+\delta \Omega_{p k}\right) t}\left\langle k_{2}\left|\Lambda_{p}\right| k\right\rangle\left\langle k\left|\Lambda_{p}^{\dagger}\right| k_{1}\right\rangle n_{p}^{(k)}(t) \\
& +\sum_{k} \frac{Z_{B}^{(k)}(t)}{Z_{B}} e^{-i \hat{\omega}_{k} t} \sum_{p} e^{-i\left(\Omega_{p}+\delta \Omega_{p k}\right) t}\left\langle k_{2}\left|\Lambda_{p}^{\dagger}\right| k\right\rangle\left\langle k\left|\Lambda_{p}\right| k_{1}\right\rangle\left(n_{p}^{(k)}(t)+1\right),
\end{aligned}
$$

where $n_{p}^{(k)}(t)=\left[\exp \left(\beta \Omega_{p}+i \delta \Omega_{p k} t\right)-1\right]^{-1}$.

The diagonal elements of the effective propagator can be partitioned into two contributions as $G_{k k}(t)=$ $G_{k k}^{0}(t)+\delta G_{k k}(t)$. Basically, $G_{k k}(t)$ reduces to $G_{k k}^{0}(t)$ when the system eigenstates are assumed to be collinear to the unperturbed states. One thus obtains

$$
G_{k k}^{0}(t)=\frac{Z_{B}^{(k)}(t)}{Z_{B}} e^{-i \hat{\omega}_{k} t}\left[1-\Lambda_{k}^{(2)}(t)\right],
$$

where $\Lambda_{k}^{(2)}(t)$ arises from the eigenstate correction that ensures their normalization up to second order as

$\Lambda_{k}^{(2)}(t)=\sum_{p}\left\langle k\left|\Lambda_{p} \Lambda_{p}^{\dagger}\right| k\right\rangle n_{p}^{(k)}(t)+\left\langle k\left|\Lambda_{p}^{\dagger} \Lambda_{p}\right| k\right\rangle\left(n_{p}^{(k)}(t)+1\right)$.
By contrast, $\delta G_{k k}(t)$ is the contribution of the propagator that involves the components of the system eigenstates which are directly coupled to the unperturbed states through the interaction $V$. These components account for a variation of the phonon number equal to unity. The contribution $\delta G_{k k}(t)$ reduces to the last two terms in Eq.(C1) for $k_{1}=k_{2}$.
* Electronic address: vincent.pouthier@univ-fcomte.fr

${ }^{1}$ V. May and O. Kuhn, Charge and Energy Transfer Dynamics in Molecular Systems (Wiley-VCH Verlag, Berlin, 2000).

${ }^{2}$ V. Agranovich, Excitations in Organic Solids (Oxford University Press, New York, 2009).

${ }^{3}$ E. R. Bittner, Quantum Dynamics: Applications in Biological and Materials Systems (Taylor and Francis/CRC Press, New York, 2009).

${ }^{4}$ H. Fröhlich, Adv. Phys. 3, 325 (1954).

5 T. Holstein, Ann. Phys. (N.Y.) 8, 325 (1959); 8, 343 (1959).

6 A. S. Davydov and N. I. Kisluka, Phys. Status Solidi B 59, 465 (1973); Zh. Eksp. Teor. Fiz 71, 1090 (1976) [Sov.
Phys. JETP 44, 571 (1976)].

7 V. Sundstrom, Prog. in Quantum Electron. 24, 187 (2000).

8 Th. Renger, V. May, and O. Kuhn, Phys. Rep. 343, 137 (2001).

9 M. Schroder, U. Kleinekathofer, and M. Schreiber, J. Chem. Phys. 124, 084903 (2006).

10 M. Mohseni, P. Rebentrost, S. Lloyd, and A. AspuruGuzik, J. Chem. Phys. 129, 174106 (2008).

11 H. Tamura, E.R. Bittner and I. Burghardt, J. Chem. Phys. 127, 034706 (2007).

12 A. Scott, Nonlinear Science: Emergence and Dynamics of Coherent Structures (Oxford University Press, New York, 2003).

13 J. Edler, R. Pfister, V. Pouthier, C. Falvo, and P. Hamm, 
Phys. Rev. Lett. 93, 106405 (2004).

14 C. Falvo and V. Pouthier, J. Chem. Phys. 123, 184709 (2005); 123, 184710 (2005).

15 D. Tsivlin and V. May, Chem. Phys. 338, 150 (2007).

16 P.A.S. Silva and L. Cruzeiro, Phys. Rev. E 74, 021920 (2006).

17 V. Pouthier, J. Chem. Phys. 128, 065101 (2008).

18 V. Pouthier and Y.O. Tsybin, J. Chem. Phys. 129, 095106 (2008).

19 E. R. Bittner, A. Goj, and I. Burghardt, Chem. Phys. 370, $137(2010)$

20 B.N.J. Persson, Phys. Rev. B 46, 12701 (1992).

21 M. Bonn M, C. Hess, and M. Wolf, J. Chem. Phys. 115, 7725 (2001).

${ }^{22}$ V. Pouthier, J.C. Light, and C. Girardet, J. Chem. Phys. 114, 4955 (2001)

23 P. Jakob, J. Chem. Phys. 114, 3692 (2001).

${ }^{24}$ V. Pouthier, J. Chem. Phys. 118, 3736 (2003).

${ }^{25}$ V. Pouthier, Phys. Rev. B 74, 125418 (2006).

${ }^{26}$ C. Gollub, Ph.D. thesis, Ludwig Maximilian University of Munich, 2009

27 M.B. Plenio, J. Hartley and J. Eisert, New J. Phys. 6, 36 (2004).

28 M. Schlosshauer, Decoherence and the Quantum-toClassical Transition (Spinger Verlag, Berlin, 2007).

29 H.P. Breuer and F. Petruccione, The theory of open quantum systems (Oxford University Press, New York, 2007).

${ }^{30}$ F. Shibata, Y. Takahashi, and N. Hashitsume, J. Stat. Phys. 17, 171 (1977).

31 C. Uchiyama and F. Shibata, Phys. Rev. E 60, 2636 (1999).

32 H.P. Breuer and B. Kappler, Annals of Physics 291, 36 (2001).

33 H.P. Breuer, J. Gemmer, and M. Michel, Phys. Rev. E 73, 016139 (2006).

34 A. Pereverzev and E. Bittner, J. Chem. Phys. 125, 104906 (2006).

35 V. Pouthier, J. Chem. Phys. 132, 035106 (2010).

36 V. Pouthier, J. Phys. : Condens. Matter 22, 255601 (2010).

37 V. Pouthier, Phys. Rev. E 81, 031913 (2010).
38 V. Pouthier, J. Phys. : Condens. Matter 22, 385401 (2010).

39 V. Pouthier, Phys. Rev. B (in press).

40 Z. Ivic, D. Kostic, Z Przulj and D Kapor, J. Phys.: Condens. Matter 9, 413 (1997).

41 D.W. Brown and Z. Ivic, Phys. Rev. B 40, 9876 (1989).

42 V. Pouthier, Phys. Rev. B 79, 214304 (2009).

43 S. Mukamel, Principles of Nonlinear Optical Spectroscopy (Oxford University Press, New York, 1995).

44 S. Bose, Phys. Rev. Lett. 91207901 (2003).

45 M. Wagner, Unitary Transformations in Solid State Physics (North-Holland, Ansterdam, 1986).

46 R. Silbey and R.W. Munn, J. Chem. Phys. 72, 2763 (1980).

47 M. Sonnek and M. Wagner, Phys. Rev. B 54, 9213 (1996).

48 V. Pouthier, J. Phys.:Condens Matter 21, 185404 (2009).

49 V. Pouthier, Phys. Rev. B 80, 144304 (2009).

50 V. Pouthier, Phys. Rev. E 78, 144304 (2008).

51 J. Wang and J. Feng, Protein Engineering 16, 799 (2003).

${ }^{52}$ R. Bloem, A. G. Dijkstra, T. la Cour Jansen, and J. Knoester, J. Chem. Phys. 129, 055101 (2008).

${ }^{53}$ K. Moritsugu, O. Miyashita, and K. Kidera, Phys. Rev. Lett. 85, 3970 (2000)

${ }^{54}$ H. Fujisaki, Y. Zhang, and J.E. Straub, J. Chem. Phys. 124, 144910 (2006)

55 N. Ostergard, in Davydov Soliton Revisited, edited by P. L. Christiansen and A. C. Scott (Plenum, New York, 1990), pp 229.

56 C. Falvo and V. Pouthier, J. Chem. Phys. 122, 014701 (2005).

57 O.V. Przhdo and P.J. Rossky, Phys. Rev. Lett. 81, 5294 (1998).

58 C.H. Bennet and D.P. DiVincenzo, Nature (London) 404, 247 (2000).

${ }^{59}$ C. M. Tesch and R. de Vivie-Riedle, Phys. Rev. Lett. 89, 157901 (2002).

${ }^{60}$ C. Tesch and R. de Vivie-Riedle, J. Chem. Phys. 121, 12158 (2004).

61 C. Gollub and R. de Vivie-Riedle, Phys. Rev. A 78, 033424 (2008) 